\title{
ON QUASICONFORMAL MAPPINGS WHICH KEEP THE BOUNDARY POINTS FIXED $\left({ }^{1}\right)$
}

\author{
BY \\ EDGAR REICH AND KURT STREBEL
}

Introduction. From a variational point of view it is natural to consider quasiconformal selfmappings of a domain which are equal to the identity on the boundary. We say that a function $\mu$ in the unit disk $U=\{z|| z \mid<1\}$ belongs to the class $\mathscr{F}$ if and only if it is the complex dilatation of a quasiconformal selfmapping $f$ of $U$ which satisfies $f\left(e^{i \theta}\right)=e^{i \theta}, 0 \leqq \theta<2 \pi$. Using the Teichmüller metric $|g(z)|^{1 / 2}|d z|$ arising from a holomorphic quadratic differential $g(z) d z^{2}$ with finite $L^{1}$-norm $\|g\|=\iint_{|z|<1}|g(z)| d x d y$ we get a necessary integral condition for $\mu \in \mathscr{F}(\S 1)$. From this inequality one easily derives a uniqueness theorem (\$3) which can be thought of as a generalization of the known result (Strebel [5]) that a Teichmüller mapping $f(\phi, k)$ which belongs to a quadratic differential $\phi$ of finite norm (i.e. has a complex dilatation $k \cdot \phi(z) /|\phi(z)|, k=$ const, $0<k<1)$ is unique extremal within the class of mappings which coincide with $f$ on $\partial U$.

Because of the importance of the class of Teichmüller mappings it is desirable to characterize those quadratic differentials for which there exists a $k>0$ such that $k(\phi /|\phi|) \in \mathscr{F}$. The last section of the present work is devoted to this question. A measurable and bounded complex valued function $\nu$ in $U$ is said to belong to the class $N$ (Ahlfors [1]) if $\iint_{U} \nu(z) g(z) d x d y=0$ for every holomorphic function $g$ in $U$ with $\|g\|<\infty$. Let $\phi$ denote a holomorphic quadratic differential in $U$, necessarily of infinite norm. It easily follows from our main inequality that if $k(\phi /|\phi|) \in \mathscr{F}$ for a sequence of positive numbers $k$ tending to zero then $\phi /|\phi| \in N$. Can one say conversely that $\phi /|\phi| \in N$ implies $k(\phi /|\phi|) \in \mathscr{F}$ ? Restricting ourselves to quadratic differentials $\phi$ of the form $\phi=\left(\Phi^{\prime}\right)^{2}$ where $\Phi$ is rational, we get a partial positive answer to this question in $\S 4$ : If $\Phi^{\prime} \neq 0$ in $U$ and $\phi /|\phi| \in N$, then $k(\phi /|\phi|) \in \mathscr{F}$ for every $k>0\left(^{2}\right)$.

\section{The main inequality.}

THEOREM 1. Let $f$ be a quasiconformal selfmapping of the unit disk $U$ which keeps the boundary fixed. Then for every holomorphic function $g$ in $U$ with finite $L^{1}$-norm the complex dilatation $\mu$ of $f$ satisfies the inequality

$$
\left|\iint_{U} \frac{\mu(z) g(z)}{1-|\mu(z)|^{2}} d x d y\right| \leqq \iint_{U} \frac{|\mu(z)|^{2}|g(z)|}{1-|\mu(z)|^{2}} d x d y
$$

Presented to the Society, April 19, 1968; received by the editors April 30, 1968.

( ${ }^{1}$ ) Work done with support from National Science Foundation grants GP-3904 and GP-7041X.

$\left({ }^{2}\right)$ Results which have in the meanwhile been obtained for the more general case when $\Phi^{\prime}$ is allowed to vanish will be presented in a future paper. 
Equality holds for $g_{0}$ iff there is $a \theta, 0 \leqq \theta<2 \pi$, such that $f$ maps every trajectory of the quadratic differential $e^{i \theta} g_{0}(z) d z^{2}$ onto itself $\left({ }^{3}\right)$.

Proof. We first discuss the case when $\zeta=G(z)=\int_{z_{0}}^{z}(g(z))^{1 / 2} d z\left(z_{0}\right.$ a fixed point of $U$ ) is single valued and, moreover, a schlicht mapping of $U$ onto a Jordan domain $U^{*}=G(U)$ in the $\zeta=\xi+i \eta$-plane. We consider the mapping $f^{*}=G \circ f \circ G^{-1}$ of $U^{*}$ onto itself. With the notation $w=f(z), d w=p d z+q d \bar{z}, w^{*}=u^{*}+i v^{*}=f^{*}(\zeta)$, $d w^{*}=p^{*} d \zeta+q^{*} d \zeta$, the length element $\left|d w^{*}\right|$ becomes

$$
\begin{aligned}
\left|d w^{*}\right| & =\left|p^{*} d \zeta+q^{*} d \zeta\right|=\left|G^{\prime}(w)\right||d w|=\left|G^{\prime}(w)\right||p d z+q d \bar{z}| \\
& =\frac{\left|G^{\prime}(w)\right|}{\left|G^{\prime}(z)\right|}\left|p d \zeta+q \frac{g(z)}{|g(z)|} d \zeta\right|
\end{aligned}
$$

which is, for the direction $d \zeta=d \xi>0$,

$$
\left|d w^{*}\right|=\left|p^{*}+q^{*}\right| d \xi=\frac{\left|G^{\prime}(w)\right|}{\left|G^{\prime}(z)\right|}\left|p+q \frac{g(z)}{|g(z)|}\right| d \xi .
$$

The various Jacobians are $J(z / \zeta)=1 /|g(z)|, J\left(w^{*} / w\right)=|g(w)|$, and therefore $J\left(w^{*} / \zeta\right)=\left|p^{*}\right|^{2}-\left|q^{*}\right|^{2}=(|g(w)| /|g(z)|)\left(|p|^{2}-|q|^{2}\right)$.

Let now $\alpha(\eta)=U^{*} \cap\{\zeta \mid \operatorname{Im} \zeta=\eta\}, \gamma(\eta)=f^{*}(\alpha(\eta))$ with Euclidean length $|\alpha(\eta)|$, $|\gamma(\eta)|$ respectively. (Interpret these lengths to be zero if $\alpha(\eta)$ is empty.) The set $\alpha(\eta)$ is a countable union of straight line segments. In view of the hypothesis on $f, \gamma(\eta)$ consists of Jordan arcs the endpoints of which coincide with the endpoints of the segments. Hence

$$
|\alpha(\eta)|=\int_{\alpha(\eta)} d \xi \leqq|\gamma(\eta)|=\int_{\alpha(\eta)}\left|p^{*}+q^{*}\right| d \xi .
$$

Integrating with respect to $\eta$ we get

$$
\|g\| \leqq \iint_{U^{*}}\left|p^{*}+q^{*}\right| d \xi d \eta=\iint_{U}\left|p^{*}+q^{*}\right||g(z)| d x d y
$$

where we have interpreted $\|g\|=\left|U^{*}\right|$ as the area of $U^{*}$. Application of the Schwarz inequality yields

$$
\begin{aligned}
\|g\|^{2} & \leqq \iint_{U} J\left(w^{*} / z\right) d x d y \cdot \iint_{U} \frac{\left|p^{*}+q^{*}\right|^{2}|g(z)|^{2}}{J\left(w^{*} / z\right)} d x d y \\
& =\|g\| \iint_{U} \frac{\left|p^{*}+q^{*}\right|^{2}}{\left|p^{*}\right|^{2}-\left|q^{*}\right|^{2}}|g(z)| d x d y \\
& =\|g\| \iint_{U} \frac{|1+\mu(z)(g(z) /|g(z)|)|^{2}}{1-|\mu(z)|^{2}}|g(z)| d x d y
\end{aligned}
$$

$\left.{ }^{3}\right)$ Every zero of $g=e^{i \alpha} g_{0}$ is then necessarily a fixed point of $f$. 
with $\mu(z)=q(z) / p(z)$. Thus

$$
\begin{aligned}
0 & \leqq \iint_{U}|g(z)|\left[\frac{|1+\mu(z)(g(z) /|g(z)|)|^{2}}{1-|\mu(z)|^{2}}-1\right] d x d y \\
& =2 \iint_{U}|g(z)| \frac{|\mu(z)|^{2}+\operatorname{Re}[\mu(z)(g(z) /|g(z)|)]}{1-|\mu(z)|^{2}} d x d y,
\end{aligned}
$$

which is equivalent to

$$
0 \leqq \iint_{U} \frac{|\mu(z)|^{2}|g(z)|+\operatorname{Re}[\mu(z) g(z)]}{1-|\mu(z)|^{2}} d x d y
$$

This holds for every $g$. Replacing $g$ by $e^{i \theta} g$ with an appropriate constant $\theta, 0 \leqq \theta<2 \pi$, we obtain (1.1).

Suppose equality holds in (1.1) for some $g_{0}$. Then there is a $\theta$ such that equality holds in (1.6) for $g=e^{i \theta} g_{0}$ and therefore in (1.2) for a.a. values of $\eta$. It follows that $\gamma(\eta)=\alpha(\eta)$ for all $\eta$. Thus $f^{*}$ maps each interval of $\alpha(\eta)$ (i.e. each trajectory of $g(z) d z^{2}$ ) onto itself, with the same orientation (because it keeps the endpoints fixed).

Conversely, let this be the case. Then $f^{*}$ is of the form $w^{*}=u^{*}(\xi, \eta)+i \eta$. We get $\left|d w^{*}\right|=u_{\xi}^{*} d \xi$ and $J\left(w^{*} / \zeta\right)=u_{\xi}^{*}$. The inequality (1.4) now reads

$$
\|g\| \leqq \iint_{U} \frac{u_{\xi}^{* 2}}{u_{\xi}^{*}}|g(z)| d x d y=\iint_{U^{*}} u_{\xi}^{*} d \xi d \eta=\|g\|
$$

Thus equality holds in (1.6) and a fortiori in (1.1).

In the general case we make use of the following facts about quadratic differentials (for the proofs see [5]). Let $I$ be an open interval on an orthogonal trajectory of $g(z) d z^{2}$. The trajectories $\alpha$ through the points of $I$ cover a simply connected domain which we call an open horizontal strip $E$. Any branch $G$ of the integral $\int(g(z))^{1 / 2} d z$ is a 1-1 conformal mapping of $E$ onto a domain $E^{*}$ in the $\zeta$-plane swept out by horizontal straight line segments through $I^{*}=G(I) . E^{*}$ has finite area $\left|E^{*}\right|=\iint_{E}|g(z)| d x d y$, and almost every trajectory in $E$ is a Jordan arc tending to a boundary point of $U$ in either direction. By a horizontal strip we mean the set of points of $U$ covered by the trajectories through a measurable subset of $I$. $U$ can be exhausted up to a set of measure zero by a sequence of nonoverlapping horizontal strips $E_{n}$. We introduce a parameter $\zeta$ in every strip $E_{n}$, i.e. we map it onto $E_{n}^{*}$ by means of a branch of $\int(g(z))^{1 / 2} d z$ in $E_{n}$. Of course we can no longer speak of the mapping $f^{*}$ in $E_{n}^{*}$, as $\int(g(z))^{1 / 2} d z$ will in general not have a single valued branch in $f\left(E_{n}\right)$, but the differentials $\left|d w^{*}\right|=|g(w)|^{1 / 2}|d w|, d u^{*} d v^{*}=|g(w)| d u d v$, and $\left|p^{*}+q^{*}\right|$, still are single valued. They represent the length and the area elements in terms of the $g$-metric in $U$.

In the definition of $\alpha(\eta)$ preceding inequality (1.2) $U^{*}$ has to be replaced by $E_{n}^{*}$, $|\alpha(\eta)|=|\alpha(\eta)|_{g}$ is the length of the corresponding trajectory of $g$ in the $g$-metric, and 
$|\gamma(\eta)|=|\gamma(\eta)|_{g}$ has to be interpreted as the $g$-length of $f(\alpha(\eta))$. The inequality is then true for every $n$, (cf. $[5, \S 9]$ ) and integration over $\eta$, followed by summation over $n$ yields

$$
\|g\| \leqq \iint_{U}\left|p^{*}+q^{*}\right||g(z)| d x d y .
$$

We then proceed as before.

In the case of equality in (1.6) we conclude from (1.2) that almost every trajectory of $g(z) d z^{2}$ is mapped onto itself by $f$, and by continuity this must be true for every trajectory. Conversely, if this is the case, $f^{*}=G \circ f \circ G^{-1}$, with $G$ a given branch of $\int(g(z))^{1 / 2} d z$, mapping $E_{n}$ onto $E_{n}^{*}$ is a selfmapping of $E_{n}^{*}$ for every $n$ and thus (1.7) becomes

$$
\|g\| \leqq \iint_{U} u_{\xi}^{*}|g(z)| d x d y=\sum_{n} \iint_{E_{n}^{*}} u_{\xi}^{*} d \xi d \eta=\|g\| .
$$

The next theorem is the analogue of Theorem 1 for quasiconformal mappings with a finite number of fixed points $z_{j}=\exp \left(i \theta_{j}\right), j=1, \ldots, n \geqq 4$, on $\partial U$. Let $g$ be a rational function which is holomorphic except for possible poles at the points $z_{j}$ and such that $g(z) d z^{2}$ is real along $\partial U-\left\{z_{j}\right\}$. This is equivalent to saying that $g(z) d z^{2}$ is a holomorphic quadratic differential with finite norm on the bordered surface $\bar{U}$, punctured at the points $z_{j}$. It can be continued by symmetry to the whole sphere $\left({ }^{4}\right)$. The set of critical trajectories (i.e. the trajectories ending at points $z_{j}$ or at zeroes of $g$ ) partitions $U$ into finitely many open "horizontal" strips $E_{n}$ which are mapped onto rectangles $E_{n}^{*}$ by $\int(g(z))^{1 / 2} d z$. The image $f(\alpha)$ of a trajectory $\alpha \subset E_{n}$ is a Jordan arc ending at the same intervals on $\partial U-\left\{z_{j}\right\}$ as $\alpha$ and therefore has length $|f(\alpha)|_{g} \geqq|\alpha|_{g}$, where the equality sign holds iff $f(\alpha)$ is a trajectory of $g$ belonging to the same strip $E_{n}$. (For more details see Strebel [5], [6].) We can thus apply the same reasoning as before. In the case of equality we conclude from (1.2) that $f$ maps every trajectory of $E_{n}$ onto another such trajectory, for every $n$. The mapping $f^{*}$ then maps $E_{n}^{*}$ onto itself and has the form $f^{*}(\zeta)=u^{*}(\xi, \eta)+i v^{*}(\eta)$. Thus $\left|d w^{*}\right|=u_{\xi}^{*} d \xi$ and $J\left(w^{*} / \zeta\right)=u_{\xi}^{*} v_{\eta}^{*}$. From the Schwarz inequality (1.4) we conclude

$$
J\left(w^{*} / z\right)=\lambda\left|p^{*}+q^{*}\right||g(z)|
$$

or equivalently

$$
J\left(w^{*} / \zeta\right)=u_{\xi}^{*} v_{\eta}^{*}=\lambda\left|p^{*}+q^{*}\right|=\lambda u_{\xi}^{*}, \quad \lambda=\text { const. }
$$

Thus $v_{\eta}^{*}=\lambda$ and therefore $v^{*}(\eta)=\lambda \cdot \eta+$ const. Evidently $\lambda$ must be equal to 1 and

$\left.{ }^{4}\right)$ The linear space of all such $g$ has real dimension $n-3$. It is most easily described after a transformation of $U$ onto the upper half plane $\operatorname{Im} \zeta>0$, which takes the points $z_{j}$ into real points $\xi_{j} \neq \infty$. We get a rational function which is real along the real axis and vanishes with at least the fourth order at infinity. 
therefore $f^{*}(\zeta)=u^{*}(\xi, \eta)+i \eta$ as before. The converse is now obvious. We have proved

THEOREM 2. Let $f$ be a quasiconformal selfmapping of $U$ which keeps $n \geqq 4$ boundary points $z_{j}$ fixed. Then for every quadratic differential $g(z) d z^{2}$ with finite norm, which is holomorphic outside the $z_{j}$ and real along $\partial U-\left\{z_{j}\right\}$ inequality (1.6) holds, with the same remarks about the equality case.

We observe that Theorem 1 continues to hold when $U$ is replaced by an arbitrary simply connected domain $\mathscr{D}$ of hyperbolic type. This follows by replacing $\mu(z)$ by

$$
\mu(z) F^{\prime}(z) / \overline{F^{\prime}(z)},
$$

and $g(z)$ by $g(z) /\left(F^{\prime}(z)\right)^{2}$, where $F$ denotes the function mapping $U$ conformally onto $\mathscr{D}$.

2. Corollaries and examples. As pointed out in the Introduction the following fact follows as an immediate corollary of Theorem 1: Suppose $\mu \in \mathscr{F}$, and $\mu$ depends on a complex parameter $t$ in such a manner that as $t \rightarrow 0$

$$
\mu(z, t)=t v(z)+o(t), \quad \sup _{z \in U}|\nu(z)|<\infty .
$$

Then $\nu \in N$. Recall that Ahlfors [1] proved that the condition $\nu \in N$ is necessary and sufficient for $\left({ }^{5}\right)$

$$
\lim _{t \rightarrow 0} \frac{f^{\mu}\left(e^{i \theta}\right)-e^{i \theta}}{t}=0, \quad 0 \leqq \theta<2 \pi .
$$

Thus our Theorem 1 can be interpreted as generalizing the necessary part of Ahlfors' condition from "small" $\mu$ to arbitrary $\mu$.

A trivial illustration of (1.1) is the following observation: If $\overline{\mu(z)}$ is analytic then either $\mu \notin \mathscr{F}$, or $\mu(z) \equiv 0$. (Proof: Put $g=\bar{\mu}$.)

Another simple application is a short proof of the following known fact [3]:

The hypothesis $\mu \in \mathscr{F}$ does not imply $t \mu \in \mathscr{F}, 0<t<1$. Namely, consider the function

$$
T_{\rho}(z)=z-(1-|z|) \rho,
$$

where $\rho$ is an arbitrary real number, $-1<\rho<1$. It is easy to verify that $T_{\rho}(z)$ is a homeomorphism $\left({ }^{6}\right)$ between the closures of $U, T_{\rho}\left(e^{i \theta}\right)=e^{i \theta}, 0 \leqq \theta<2 \pi$, and hence the complex dilatation

$$
\mu(z)=\frac{\partial T_{\rho}}{\partial \bar{z}} / \frac{\partial T_{\rho}}{\partial z}=\frac{\rho z}{2|z|+\rho \bar{z}}
$$

( $\left.{ }^{5}\right) f^{u}(z)$ denotes the quasiconformal mapping of $U$ onto $U$ with complex dilatation $\mu(z)$, normalized so that $f^{\mu}$ keeps the points $1, i,-1$ fixed.

$\left.{ }^{(}\right)$The mapping $T_{\rho}(z)$ shifts the point 0 to $-\rho$. In fact, for small $\rho, T_{\rho}(z)$ approximates the Teichmüller shift function [7] within an error $o(\rho)$. 
belongs to the class $\mathscr{F}$. On the other hand, if $g(z)$ is holomorphic in $U$, and $\|g\|<\infty$, then

$$
I=\iint_{U} \mu(z) g(z) d x d y=\int_{0}^{1} r d r \int_{|z|=r} g(z) \frac{\rho z}{2 r+\rho r^{2} / z} \frac{d z}{i z}=-\frac{\pi}{2} \int_{0}^{1} r g\left(-\frac{\rho r}{2}\right) d r .
$$

Choosing, say $\rho=\frac{1}{2}, g(z) \equiv 1$, we obtain $I \neq 0$; i.e. $\mu \notin N$. Therefore, $t \mu \notin \mathscr{F}$ for all sufficiently small $t$.

The example

$$
\mu(z)=t z, \quad(0<t<1)
$$

shows that (1.1) is not sufficient to guarantee that $\mu \in \mathscr{F}$. For, evaluating the left side of (1.1) in polar coordinates we obtain the value 0 . On the other hand, we will now verify that $\mu \notin \mathscr{F}$. The function $w^{*}=Q(z)=z \exp (t \bar{z}), z \in U$, is a quasiconformal function with complex dilatation $t z$. Since $\arg Q\left(e^{i \theta}\right)$ is monotonic, $Q$ is actually a homeomorphism. Let $\zeta=\Psi(w)$ map $U$ conformally onto $Q(U)$, normalized in such a manner that $f^{\mu}(z)=\Psi^{-1} \circ Q(z)$. Since $\partial Q(U)$ is an analytic curve, $\Psi(w)$ is analytic in a neighborhood of, say, $w=1$. Suppose now that $f^{\mu}(z) \equiv z$, $|z|=1$. Then

$$
\Psi(z)=z \exp (t \bar{z})=z \exp (t / z), \quad|z|=1 .
$$

By the uniqueness of analytic extension it follows that $\Psi(z)=z \exp (t / z), z \in U-\{0\}$, an evident impossibility as $\Psi(z)$ must have an analytic extension to all of $U$, and thus cannot have a singularity at $z=0$.

The above example (2.2) also provides an illustration of the fact that it is possible to have $\mu \in N$, and at the same time $t \mu \notin \mathscr{F}, 0<t<1$.

3. A uniqueness theorem. Motivated by the known variational techniques for quasiconformal mappings ([2], [4]) we can produce variations within the class of mappings of $U$ onto itself with a specified boundary correspondence by composing one such mapping with mappings which keep the boundary points fixed. Theorem 1 then imposes a certain restriction on the complex dilatation of the composed mapping which in certain cases may serve to identify an extremal function. As an illustration of this technique we prove the following extremal and uniqueness theorem. It generalizes [5] in the sense that the absolute value of the complex dilatation $\lambda$ of the extremal mapping $h$ need not be constant. The interesting point is that it is the inverse mapping $h^{-1}$ which is associated with a quadratic differential; in the special case $|\lambda|=k=$ const it makes no difference whether we consider $h$ or $h^{-1}$, as is easily seen.

THEOREM 3. Let $h$ and $\tilde{h} \neq h$ be quasiconformal selfmappings of $U$ which coincide on $\partial U$, and denote by $\lambda$ and $\tilde{\lambda}$ their complex dilatations. If the complex dilatation $\kappa$ of the inverse $h^{-1}$ of $h$ is of the form $\kappa(w)=k(w)(\bar{\psi}(w) /|\psi(w)|)$ with $k(w) \geqq 0$ and $\psi$ holomorphic with finite norm, then $|\tilde{\lambda}(z)|>|\lambda(z)|$ on a set of positive measure. 
Proof. Let $f=\tilde{h} \circ h^{-1}$ and denote the complex dilatation of $f$ by $\mu$. Expressing $\tilde{\lambda}$ in terms of $\mu$ and $\lambda$ we get

$$
\tilde{\lambda}(z)=\frac{\lambda(z)+\mu(w) \tau(z)}{1+\lambda(z) \mu(w) \tau(z)}
$$

with

$$
\tau(z)=\overline{h_{z}(z)} / h_{z}(z), \quad w=h(z) .
$$

Assume $|\tilde{\lambda}(z)| \leqq|\lambda(z)|$ a.e. in $U$. A short computation shows that this inequality is equivalent to

$$
|\mu(w)|^{2}\left(1+|\lambda(z)|^{2}\right)+2 \operatorname{Re}[\bar{\lambda}(z) \mu(w) \tau(z)] \leqq 0 .
$$

The complex dilatation $\kappa$ of $h^{-1}$ expressed by means of $\lambda$ is $\kappa(w)=-\lambda(z) \bar{\tau}(z)$, which yields

$$
|\mu(w)|^{2}\left(1+|\kappa(w)|^{2}\right)-2 \operatorname{Re}[\bar{\kappa}(w) \mu(w)] \leqq 0 .
$$

Making use of the hypothesis we get

$$
|\psi(w)||\mu(w)|^{2}\left(1+k^{2}(w)\right)-2 k(w) \operatorname{Re}[\mu(w) \psi(w)] \leqq 0 .
$$

Let $E_{0}$ be the set of points $w$ with $k(w)=0$. On $E_{0}$ we conclude from (3.4) that $\mu(w)=0$. Therefore this set gives no contribution to the integral (1.1). For almost all points $w \in U-E_{0}$ we have $0<k(w) \leqq k_{0}, k_{0}=\sup _{w \in U} k(w)<1$, and thus

$$
\left(1+k^{2}(w)\right) / 2 k(w) \geqq\left(1+k_{0}^{2}\right) / 2 k_{0}=1+\left(1-k_{0}\right)^{2} / 2 k_{0} .
$$

Dividing (3.4) by $2 k(w)\left(1-|\mu(w)|^{2}\right)$ and applying (3.5) gives

$$
\frac{|\psi(w)||\mu(w)|^{2}}{1-|\mu(w)|^{2}}-\frac{\operatorname{Re} \mu(w) \psi(w)}{1-|\mu(w)|^{2}}+\frac{\left(1-k_{0}\right)^{2}}{2 k_{0}} \frac{|\psi(w)||\mu(w)|^{2}}{1-|\mu(w)|^{2}} \leqq 0 .
$$

Integration over $U-E_{0}$ and application of (1.1) in the version (1.6) with $g=-\psi$ finally yield

$$
\frac{\left(1-k_{0}\right)^{2}}{2 k_{0}} \iint_{U-E_{0}} \frac{|\psi(w)||\mu(w)|^{2}}{1-|\mu(w)|^{2}} d u d v \leqq 0
$$

and therefore $\mu(w)=0, w \in U-E_{0}$. Q.E.D.

In exactly the same way Theorem 2 can be applied to prove the following

THEOREM 4. Suppose we are given finitely many points $z_{j} \in \partial U, j=1, \ldots, n \geqq 4$, and a quasiconformal selfmapping $h$ of $U$, the inverse $h^{-1}$ of which has a complex dilatation $\kappa(w)=k(w)(\bar{\psi}(w) /|\psi(w)|), k(w) \geqq 0$, where $\psi$ is a holomorphic quadratic differential of $\bar{U}-\left\{z_{j}\right\}$ with finite norm. If $\tilde{h}$ is a quasiconformal selfmapping of $U$ with complex dilatation $\tilde{\lambda}$ such that $\tilde{h}\left(z_{j}\right)=h\left(z_{j}\right),|\tilde{\lambda}(z)| \leqq|\lambda(z)|$ a.e. in $U$, then $\tilde{h}=h$.

Proof. The quadratic differentials mentioned in Theorems 2 and 4 are of the same nature. It is thus again possible to put $g=-\psi$ in the main inequality. 
Further generalizations, where some intervals on $\partial U$ or even an arbitrary closed point set is kept fixed are of course possible.

4. Some remarks on Teichmüller mappings which keep the boundary fixed. Let $f(\phi, k)$ be a Teichmüller mapping which is equal to the identity on $\partial U$. The complex dilatation of $f$ is $\mu(z)=k \cdot \phi(z) /|\phi(z)|$. Introducing this expression in (1.1) we get

$$
\left|\iint_{U} \frac{\phi(z)}{|\phi(z)|} g(z) d x d y\right| \leqq k\|g\| .
$$

If a given holomorphic quadratic differential $\phi$ admits a sequence of values $k_{n} \rightarrow 0$ $\left(k_{n}>0\right)$ such that $f\left(\phi, k_{n}\right)$, when normalized at three points on $\partial U$, keeps the boundary fixed, we get

$$
\iint_{U} \frac{\phi(z)}{|\phi(z)|} g(z) d x d y=0
$$

for every holomorphic function $g$ which has finite norm in $U$. Such a $\phi$ must clearly satisfy the necessary condition

$$
\|\phi\|=\infty,
$$

as otherwise (4.2), with $g(z)=\phi(z)$, would imply $\phi(z) \equiv 0$. We now make the further restriction $\phi=\Phi^{\prime 2}, \Phi$ rational. Equality (4.2) then reads

$$
\iint_{U} \frac{\overline{\Phi^{\prime}}(z)}{\Phi^{\prime}(z)} g(z) d x d y=0 .
$$

The next theorem gives a characterization of the class of functions $\Phi$ satisfying (4.4).

THEOREM 5. A nonconstant rational function $\Phi$ which is holomorphic in $U$ satisfies the orthogonality relation

$$
\iint_{U} \frac{\phi}{|\phi|} g d x d y=0, \quad \phi=\Phi^{\prime 2}
$$

for every $g$ which is holomorphic in $U$ and has finite norm $\|g\|=\iint_{U}|g(z)| d x d y$ if and only if the following conditions hold.

(a) All the poles of $\Phi$ are on $|z|=1$.

(b) If $\Phi(z)-\Phi\left(z_{0}\right)$ has a zero of multiplicity $m(m \geqq 2)$ at $z_{0}\left(\left|z_{0}\right|<1\right)$ then $\Phi(z)$ $-\Phi\left(z_{0}\right)$ has a zero of multiplicity at least $m-1$ at $z=1 / \bar{z}_{0}$. (If $\Phi^{\prime}\left(z_{0}\right) \not \equiv 0$, this condition is of course void.)

Proof. (1) Let $R(z)=(\bar{\Phi}(z)-\bar{\Phi}(1 / \bar{z})) / \Phi^{\prime}(z)$. We have $R_{\bar{z}}(z)=\phi(z) /|\phi(z)|$ and therefore

$$
\begin{aligned}
\iint_{|z|<1} \frac{\phi(z)}{|\phi(z)|} g(z) d x d y & =\frac{1}{2 i} \iint_{|z|<1} \frac{\partial}{\partial \bar{z}}(R g) d \bar{z} d z \\
& =\frac{1}{2 i} \lim _{r \rightarrow 1} \int_{|z|=r} R g d z-\frac{1}{2 i} \sum_{k} \lim _{\rho \rightarrow 0} \int_{\left|z-z_{k}\right|=\rho} R g d z,
\end{aligned}
$$


where the $z_{k}$ are the singularities of $R$, i.e. $\left|z_{k}\right|<1,1 / \bar{z}_{k}$ a pole of $\Phi$, or $z_{k}$ a zero of $\Phi^{\prime}$. The limits evidently exist because of the absolute convergence of the integral $\iint_{|z|<1} R_{z} g d x d y$ and Green's theorem.

We first show that

$$
\lim _{r \rightarrow 1} \int_{|z|=r} R(z) g(z) d z=0 .
$$

It follows from $\iint\left|g\left(r e^{i \theta}\right)\right| r d r d \theta<\infty$ that there exists a sequence $r_{n} \rightarrow 1$ such that $\left(1-r_{n}\right) \int\left|g\left(r_{n} e^{i \theta}\right)\right| r_{n} d \theta \rightarrow 0$. The result then follows from $R(z)=O(1-r)$ for $r \rightarrow 1$. To this end we write $R(z) /(1-r)=R(z)(1+r) /(1-z \bar{z})$ and show that to every point $\zeta,|\zeta|=1$, there exists a neighborhood $U(\zeta)$ and a bound $M$ such that $|R(z)| /(1-z \bar{z})<M$ for $z \in U(\zeta)$. We can then $\operatorname{cover}\{|z|=1\}$ with finitely many such neighborhoods, choose the largest bound $M$ and $r_{0}<1$ such that for $r_{0}<|z|<1$ the point $z$ is in at least one of the neighborhoods. Let $\zeta,|\zeta|=1$, be a regular point of $\Phi$. In some neighborhood of $\zeta \Phi$ has an expansion

$$
\Phi(z)=a_{0}+(\bar{z}-\zeta)^{m}\left(a_{m}+a_{m+1}(z-\zeta)+\cdots\right), \quad\left(a_{m} \neq 0, m \geqq 1\right) .
$$

Thus

$$
\frac{R(z)}{1-z \bar{z}}=\frac{\bar{a}_{m}\left[(\bar{z}-\bar{\zeta})^{m}-\left(\frac{1}{z}-\zeta\right)^{m}\right]+\bar{a}_{m+1}\left[(\bar{z}-\zeta)^{m+1}-\left(\frac{1}{z}-\zeta\right)^{m+1}\right]+\cdots}{(1-z \bar{z})(z-\zeta)^{m-1}\left(m a_{m}+\cdots\right)} .
$$

The general term in the numerator reads

$$
\bar{a}_{m+j} \frac{z \bar{z}-1}{z}\left[(z-\bar{\zeta})^{m+j-1}+(\bar{z}-\xi)^{m+j-2}\left(\frac{1}{z}-\xi\right)+\cdots+\left(\frac{1}{z}-\xi\right)^{m+j-1}\right]
$$

which, since $|1 / z-\xi|=|(\zeta-z) / \zeta z|=|\zeta-z| /|z|$, has the upper bound

$$
\left|a_{m+j}\right| \frac{1-z \bar{z}}{|z|^{m}}|z-\zeta|^{m-1}(m+j)\left(\frac{|z-\zeta|}{|z|}\right)^{j}, \quad j=0,1,2, \ldots
$$

Thus for sufficiently small $|z-\zeta|$ we have a finite bound

$$
\frac{|R(z)|}{1-z \bar{z}} \leqq \frac{2}{m\left|a_{m}\right|} \frac{1}{|z|^{m}} \sum_{j=0}^{\infty}\left|a_{m+j}\right| \cdot(m+j)\left(\frac{|z-\zeta|}{|z|}\right)^{j} \leqq M .
$$

Now let $\zeta$ be a pole of $\Phi$ of order $n \geqq 1$ :

$$
\Phi(z)=\frac{a_{-n}}{(z-\zeta)^{n}}+\cdots+\frac{a_{-1}}{z-\zeta}+a_{0}+\cdots, \quad\left(a_{-n} \neq 0\right) .
$$

We have

$$
\frac{R(z)}{1-z \bar{z}}=\frac{\bar{a}_{n}\left[\frac{1}{(\bar{z}-\zeta)^{n}}-\frac{1}{((1 / z)-\zeta)^{n}}\right]+\cdots}{(1-z \bar{z})\left(1 /(z-\zeta)^{n+1}\right)\left[-n a_{-n}+\cdots\right]}
$$


The general singular term of the numerator has the form

$$
\bar{a}_{-n+j} \frac{\zeta(1-z \bar{z})}{(\bar{z}-\zeta)(\zeta-z)}\left[\frac{1}{(\bar{z}-\zeta)^{n-j-1}}+\frac{1}{(\bar{z}-\zeta)^{n-j-2}} \cdot \frac{1}{((1 / z)-\zeta)}+\cdots\right]
$$

and has upper bound

$$
\left|a_{-n+j}\right| \frac{1-z \bar{z}}{|\zeta-z|^{2}} \frac{n-j}{|z-\zeta|^{n-j-1}}, \quad j=0, \ldots, n-1 .
$$

For the regular terms we get an upper bound similar to the one before, which completes the proof of (4.5). Therefore, for any $g$ with $\|g\|<\infty$ we have

$$
\iint_{|z|<1} R_{\bar{z}} g d x d y=\frac{1}{2 i} \sum_{k} \lim _{\rho \rightarrow 0} \int_{\left|z-z_{k}\right|=\rho} R g d z .
$$

(2) Let us now consider the term $A_{k}=\lim _{\rho \rightarrow 0} \int_{\left|z-z_{k}\right|=\rho} R g d z$ for arbitrary $z_{k}$. Making use of polynomials of the form $g(z)=\prod_{k}\left(z-z_{k}\right)^{n_{k}}$ we first derive necessary conditions such that $\sum_{k} A_{k}=0$ for every $g$. At $z_{k} \Phi$ has an expansion

$$
\Phi(z)=a_{0}+\left(z-z_{k}\right)^{m}\left(a_{m}+a_{m+1}\left(z-z_{k}\right)+\cdots\right)
$$

where $a_{m} \neq 0$ and $m \geqq 1$ is the multiplicity of $a_{0}=\Phi\left(z_{k}\right)$. Assume first that $\Phi$ has a pole of order $n \geqq 1$ at the mirror point $1 / \bar{z}_{k}$ :

$$
\dot{\Phi}(z)=\frac{b_{-n}}{\left(z-1 / \bar{z}_{k}\right)^{n}}+\cdots, \quad b_{-n} \neq 0, \text { near } 1 / \bar{z}_{k} .
$$

Then

$$
R(z)=\frac{\bar{a}_{0}+\left(\bar{z}-\bar{z}_{k}\right)^{m}\left(\bar{a}_{m}+\cdots\right)-\left(z z_{k} /\left(z_{k}-z\right)\right)^{n}\left(\bar{b}_{-n}+\cdots\right)}{\left(z-z_{k}\right)^{m-1}\left(m a_{m}+\cdots\right)}
$$

If we choose the factor $\left(z-z_{k}\right)^{n_{k}}$ of $g$ with $n_{k}=m+n-1$, this point $z_{k}$ will give no contribution to the sum $\sum_{k} A_{k}$, whereas, if we choose $n_{k}=m+n-2$ its contribution will be different from zero. Therefore, if $\Phi$ has a pole outside $|z| \leqq 1$ (for a pole at infinity the computation is similar) we can choose a function $g$ such that

$$
\iint_{|z|<1} R_{z} g d x d y \neq 0 .
$$

We have proved the necessity of the condition (a).

To prove the necessity of (b) let the development of $\Phi(z)$ at $1 / \bar{z}_{k}$ be

$$
\Phi(z)=b_{0}+\left(z-1 / \bar{z}_{k}\right)^{l}\left(b_{l}+\cdots\right), \quad b_{l} \neq 0 .
$$

We have

$$
R(z)=\frac{\bar{a}_{0}+\left(\bar{z}-\bar{z}_{k}\right)^{m}\left(\bar{a}_{m}+\cdots\right)-\left[\bar{b}_{0}+\left(\left(z_{k}-z\right) / z z_{k}\right)^{l}\left(\bar{b}_{l}+\cdots\right)\right]}{\left(z-z_{k}\right)^{m-1}\left(m a_{m}+\cdots\right)}
$$

Choosing $n_{k}=m-1$ as exponent of the factor $z-z_{k}$ of $g$ the integral vanishes in the limit; if $m \geqq 2$ and we choose $n_{k}=m-2$, the integral only vanishes if $a_{0}=b_{0}$. Let this 
be the case. Then the first remaining term in the numerator does not give a contribution in the limit, for any $g$. If $l \leqq m-2$, we choose $n_{k}$ such that $n_{k}+l-(m-1)$ $=-1$, i.e. $n_{k}=m-2-l \geqq 0$ to get a nonvanishing contribution. This shows the necessity of (b).

(3) Now let conditions (a) and (b) be satisfied. Then at an arbitrary zero $z_{k}$ of $\Phi^{\prime}$, $\left|z_{k}\right|<1$, we have the above expansion with $a_{0}=b_{0}$ and $l \geqq m-1$. But then for arbitrary holomorphic $g$ evidently $\lim _{\rho \rightarrow 0} \int_{\left|z-z_{k}\right|=\rho} R g d z=0$. This completes the proof.

We will now see that if $\Phi$ satisfies the hypothesis of Theorem 5 , and $\Phi^{\prime}(z) \neq 0$ in $U$, a much stronger conclusion follows.

THEOREM 6. Let $\Phi \neq$ const be a rational function which is holomorphic in the extended plane except possibly on $\{|z|=1\}$ and has a nonvanishing derivative when $|z|<1$. Then for every $k, 0<k<1$, the normalized Teichmüller mapping $f(\phi, k)$, $\phi=\Phi^{\prime 2}$ of $U$ onto $U$ keeps the boundary fixed.

Proof. After a linear transformation we may assume that $U$ is the upper half plane $\{z=x+i y \mid y>0\}$ and $\Phi$ has the form

$$
\Phi(z)=\sum_{j=1}^{n} H_{j}(z), \quad H_{j}(z)=\sum_{\nu=0}^{n_{j}} \frac{a_{j v}+i b_{j v}}{\left(z-x_{j}\right)^{v}}
$$

$x_{j}, a_{j v}, b_{j v}$ real. We consider the function

$$
\Psi(z)=\sum_{j=1}^{n} H_{j}^{*}(z), \quad H_{j}^{*}(z)=\sum_{v=0}^{n_{j}} \frac{K a_{j v}+i b_{j v}}{\left(z-x_{j}\right)^{v}}, \quad K=\frac{1+k}{1-k} .
$$

For $z=x \neq x_{j}$ we have

$$
\operatorname{Im} \Psi(x)=\operatorname{Im} \Phi(x), \operatorname{Re} \Psi(x)=K \operatorname{Re} \Phi(x) .
$$

Let $x_{0} \neq x_{j}$. If the power series for $\Phi$ is

$$
\Phi(z)=\left(z-x_{0}\right)^{l}\left(\left(A_{l}+i B_{l}\right)+\left(A_{l+1}+i B_{l+1}\right)\left(z-x_{0}\right)+\cdots\right)
$$

the power series for $\Psi$ at the same point must be

$$
\Psi(z)=\left(z-x_{0}\right)^{l}\left(\left(K A_{l}+i B_{l}\right)+\left(K A_{l+1}+i B_{l+1}\right)\left(z-x_{0}\right)+\cdots\right),
$$

because for $z=x$ this is the only power series which satisfies (4.8). The radius of convergence of both series is the same. Let $F$ denote a horizontal stretching by a factor $K$. Every point $x_{0}$ on the closed real axis, including the points $x_{j}$ and the zeroes of $\Phi^{\prime}$, has a circular neighborhood $V$ in which $\Psi^{-1} \circ F \circ \Phi$, with $\Psi^{-1}$ a properly chosen branch, is single valued and schlicht, and keeps the points on the real axis fixed. In the intersection of two such neighborhoods the two mappings coincide. Thus a sufficiently large circle $\gamma$ in the upper half plane which intersects a small neighbourhood of the origin is mapped by $w=\Psi^{-1} \circ F \circ \Phi(z)$ onto a Jordan curve $\gamma^{\prime}$ in $\{\operatorname{Im} w>0\}$. As $\Phi^{\prime}(z) \neq 0$ in $\{\operatorname{Im} z>0\}$ :

$$
\frac{1}{2 \pi} \int_{\gamma} d \arg \Phi^{\prime}=\frac{1}{2 \pi} \int_{\gamma}(d \arg (d \Phi)-d \arg (d z))=0 ;
$$


thus $\int_{\gamma} d \arg (d \Phi)=2 \pi$, where $d \Phi$ is the tangent vector of the $\Phi$-image of $\gamma$. The winding number of the tangent vector of the curve $F \circ \Phi(\gamma)$ evidently is the same (by continuity, letting $K$ increase from one to its final value). Thus $\int_{\gamma^{\prime}} d \arg (d \Psi)$ $=2 \pi$ and we conclude that $\Psi^{\prime \prime}$ has no zero inside $\gamma^{\prime}$ and by exhaustion in the entire upper half plane.

Now $F \circ \Phi$ maps the interior of $\gamma$ onto a covering surface $R$ which (considered e.g. as a surface lying over the plane) is spanned by the curve $F \circ \Phi(\gamma)$ and which has no branching. The same is true for $\Psi$ and the interior of $\gamma^{\prime}$, with $\Psi\left(\gamma^{\prime}\right)=F \circ \Phi(\gamma)$. As may be verified there is only one such covering surface $R$, and from this we conclude that the boundary mapping $\Psi^{-1} \circ F \circ \Phi$ of $\gamma$ onto $\gamma^{\prime}$ can be extended to a quasiconformal mapping of the disk $D=\operatorname{Int} \gamma$ onto Int $\gamma^{\prime}$. Expanding $D$ to the upper half plane we get the result that $\Psi^{-1} \circ F \circ \Phi$ maps $U$ onto $U$ keeping every point on the real axis fixed.

EXAMPLES. If the restriction of $\Phi(z)$ to $U$ is schlicht, then the restriction of $\Psi(w)$ to $U$ is also schlicht, and the poles can be at most second order. A trivial example is obtained when $\Phi(U)$ is the right half plane, or the plane slit along the positive real axis. In this case $F \circ \Phi(U)=\Phi(U)$.

To consider a less obvious example let $\Phi(z)=(z-1) /(z+1)^{2}$, which is schlicht in $U$, and maps $U$ onto the "exterior" of a parabola. The affine mapping stretches the parabola, but the mapping $\Psi(w)$ depends on $k$ in such a manner that the composed mapping $\Psi^{-1} \circ F \circ \Phi$ keeps all points of $\{|z|=1\}$ fixed.

REMARK. For complex dilatations $\mu=k \phi /|\phi|$ where $\phi$ is of the form considered in Theorem 6 it is clear, in contrast with $\left(2.1^{\prime}\right)$, that not only is $\mu \in \mathscr{F}$ but also $t \mu \in \mathscr{F}, 0<t<1$.

\section{REFERENCES}

1. Lars V. Ahlfors, Some remarks on Teichmüller's space of Riemann surfaces, Ann. of Math. 74 (1961), 171-191.

2. P. P. Belinskir, The use of a variational method in solving problems of quasiconformal mappings, Dokl. Akad. Nauk SSSR 121 (1958), 199-201.

3. F. W. Gehring, Quasiconformal mappings which hold the real axis pointwise fixed, Macintyre memorial volume. Ohio University Press, Athens (to appear).

4. M. Schiffer, A variational method for univalent quasiconformal mappings, Duke Math. J. 33 (1966), 400-411.

5. Kurt Strebel, Zur Frage der Eindeutigkeit extremaler quasikonformer Abbildungen des Einheitskreises. II, Comment. Math. Helv. 39 (1964), 77-89.

6. - On quadratic differentials and extremal quasiconformal mappings, lecture notes, University of Minnesota, Minneapolis, 1967.

7. Oswald Teichmüller, Ein Verschiebungssatz der quasikonformen Abbildung, Deutsche Math. 7 (1944), 336-343.

UNIVERSITY OF MINNESOTA, MinNEAPOLIS, MinNESOTA

UNIVERSITY OF ZÜRICH, ZÜRICH, SWITZERLAND 